\title{
2D Convolution Operation with Partial Buffering Implementation on FPGA
}

\author{
Arun Mahajan \\ CEC Landran, Mohali/ECE Dept, Chandigarh, 140307, India \\ Email: arunnmahajann@gmail.com \\ Mr. Paramveer Gill \\ CEC Landran, Mohali/ECE Dept, Chandigarh, 140307, India \\ Email: paramveer.ece@cgc.edu.in
}

\begin{abstract}
In the modern digital systems, the digital image processing and digital signal processing application form an integrated part in the system design. Many designers proposed and implemented various resources and speed efficient approaches in the recent past. The important aspect of designing any digital system is its memory efficiency. The image consists of various pixels and each pixel is again holds a value from 0 to 255 which requires 8 bits to represent the range. So a larger memory is required to process the image and with the increase in size of the image the number of pixels also increases. A buffering technique is used to read the pixel data from the image and process the data efficiently. In the work presented in this paper, different window sizes are compared on the basis of timing efficiency and area utilization. An optimum window size must be selected so as to reduce the resources and maximize the speed. Results show the comparison of various window operations on the basis of performance parameters. In future other window operation along with convolution filter like Adaptive Median filter must be implemented and used by changing the row and column values in Window size.
\end{abstract}

Index Terms-2D Convolution, Median Filter, FPGA

\section{INTRODUCTION}

Image de-noising is the process to eliminate the noise from the images. Nonlinear filter such as adaptive mean filter are used for image de-noising because they reduce the smoothing and preserve the image edges. Digital images are affected by the noise formed from transmission of images, acquisition, scanner, camera sensor and many more. Noise in the image basically refers to change in the display of image such as contrast, brightness, etc. usually, noise can be defined as erroneous intensity variation that take place generally, because of imperfections in imaging device that utilized to achieve images or in other words, because of interruption in transmission channels [9]. While capturing image there is a chance of inclusion of noise, which will affect the pixel intensity values. There are a plenty sources of noise that affects the image. Some sources included transmission errors, imperfect instruments, natural phenomena, and imperfect data acquisition process and compression techniques. The image noise may not be visible but will be there in the image. The image quality is affected by many factors such as environmental temperature, sensitivity of camera, time taken to capture images and so on. The brightness, color, smoothness in the image gets affected thus producing a picture, which is undesirable. The following image is a noisy image with the presence of excessive random noise [3]. There are several kinds of noise from which images are influenced. Some of these noises are gamma noise, uniform and non-uniform noise, Gaussian noise and many more. In the digital images, impulse noise is one of the major sources of error. This type of noises is taking place through image transmission. Elimination of noise usually refers to filter the signal that can have more or less noise, which affects the image quality. The major objective of de-noising is to improve the real image while eliminating the noisy pixel [4].

The median filter algorithms optimize the sorting process to reduce the computational complexity. The median filter algorithms increase the quality of the filtered images without increasing the computational complexity of median filter algorithm. These algorithms try to detect the noisy pixels and adaptively filter only these noisy pixels. However, the adaptive median filter algorithm presented in this work both reduces the computational complexity of median filter algorithm and increases the quality of filtered images by exploiting the pixel correlations in the input image[10].

\section{RELATED WORK}

Verma, Kesari,et al. [1]in this paper, improvement in Adaptive Median Filter has been presented used for preservation of edges. In biomedical images edges are the significant feature. The major objective of this work is to preserve the edges without losing in peak signal to noise ratio and SNR. In this work, a novel constraint for calculating Edge Retrieval Index (ERI) has also been proposed which helps to evaluate the edge preservation index in images.in homogenous area, proposed approach cleans the entire image noise but preserves the edges. The results indicate that the proposed approach can be 
adapted to eliminate noise and prevent edges. The result also proves that, by using this approach edge lost is minimal.

Saleem, S. Abdulet al. [2] in this study, an Effective Noise Adaptive Median Filter have been proposed in order to eliminate Impulse Noises from Color Images. Color images are degraded by noise due to transmission, acquisition and storage. This proposed method helps to remove impulse noise and also maintained its image details. This method also helps to enhance its image quality. This novel method uses a spatial domain approach and $3 \times 3$ overlapping window to filter the signal. The proposed median filter has been assessed using MATLAB and simulations have been done on a both gray scale and color images. The results demonstrated the effectiveness of median filter when compared with some other adaptive mean algorithm.

Chakravarthy, SR Sannasi et al. [3] proposed a boundary discriminative noise detection algorithm for eliminating impulse noise and random noise. The features of Image will degrade by the impact of noise. In this work, modifications to the filtering step of the BDND algorithm has been presented by increasing the window size one step higher to existing size to solve those issues. The results indicate that proposed boundary discriminative noise detection algorithm can produce sharper image and also noise is removed. This presented algorithm has been implemented using MATLAB 7.12 using image-processing toolbox.

Kaur Amanpreet, Rahul Malhotra et al. [4] presented a non-iterative adaptive median filter. In this work, noisy pixel is removed from given window using proposed method i.e. non-iterative median filter. The proposed approach indicates efficient performance and it helps to eliminate the noisy pixel. In order to propose the median filters in MATLAB, Image processing toolbox has been used.

Habib, MuhammadNoiseet al. [5] a adaptive fuzzy inference system has been presented in this paper. This novel approach is used for arbitrary value impulse noise recognition and elimination. The presented filter utilizes the intensity, which depends on statistics to build adaptive fuzzy membership functions. The Simulation results are depending upon PSNR indicates the efficiency of presented filter.

Bhateja, Vikrantet al. [6] proposed a non-iterative adaptive median filter for removing noise infected with impulse noise. This scheme works in 2 stages. The presented process is verified on the images having distinct features and it may be seen that it is generating good results in terms of qualitative and quantitative measures of the image in contrast to another filtering methods.

Meher, SarojKet al. [7] in this paper, enhanced recursive and adaptive median filter has been presented. Proposed RAMF method used for the re-establishment of images tarnished with impulse noise. The size of window may change, depending upon the occurrence of noise free pixels. The performance indicates that the proposed approach is extremely efficient as compared with other algorithm in term of PSNR and image enhancement factor. The dominance of the proposed filter is also necessary by visual analysis.

Shanmugavadivuet al [8] in this work, a novel noise filter has been proposed which helps to eliminate noise from the image, which is tarnished, by impulsive noise. The performance of presented filter is better as compare to other traditional approaches in term of peak SNR. The presented filter demonstrated that it is very efficient for eliminating noise from the images which having $90 \%$ of noise.

Mukherjee, Manaliet al. [9] this work proposed low complexity reconfigurable hardware architecture. Adaptive Median filter has been proposed in this work for eliminating the noise from the digital images. MSE and PSNR have been done in order to contrast the performance of median and adaptive median filter. This work presented a hardware implementation that is necessary for real time execution. FPGA is highly utilized for the processing in real time. The simulation result has been done using the Xilinx ISE 14.5 software.

Kalali, Ercan, et al[10] Presented a 2D adaptive median filter algorithm in this paper. In this work adaptive median filter reduces the complexity of $2 \mathrm{D}$ filters and it gives good quality images. The novel methods have been implemented for $5 * 5$ window size. the proposed hardware can process 105 full HD (1920x1080) images per second in the worst case on a Xilinx Virtex 6 FPGA, and it has more than $80 \%$ less energy consumption than original $2 \mathrm{D}$ median filter hardware on the same FPGA.

Hsieh, Mu-Hsien et al [11] This novel approach is used for random valued impulse noise detection and removal. The proposed median filter has been assessed using MATLAB and simulations have been done on a both gray scale and color images. The results indicated that effectiveness of median filter when compared with some other adaptive mean algorithm. The presented approach re established noisy images with 1-99\% levels of salt-and-pepper impulse noise.

Sree, P. Syamala Jayaet. al [12] presented a novel adaptive median-based lifting filter. This is used to eliminate noise from the images by computing the median of neighboring pixel. In this paper, proposed algorithm uses lifting scheme to remove the noise. The numerical result indicates that the proposed algorithm is very efficient and this filter is compared with other traditional filters. On the basis of comparison it is found that the proposed method performs well and it can eliminate salt and pepper noise as high as $90 \%$. This method works exceptionally well at each level of noise in terms of PSNR and SSIM.

Deka, Bhabesh et al[13] presented multi-scale based adaptive median filter which has ability to re establish images degraded by impulsive and salt-pepper noise and having very high noise ratios. The propose approach is efficient, simple and easy to implement. In this paper, noise detection method is used depending on the multiscale filtering and switching median filter. Several switching filter has been discussed in this paper such as 
DWM, SDROM, BDND, ACWM and many more. These filers are compared and evaluated with the proposed adaptive filter. And it is found that proposed adaptive filter is better than other existing methods. this proposed method also helps to preserve the textures details and edge of images.

Zhang, Peixuanet al[14] in this paper, a novel adaptive weighted mean filter (AWMF) has been presented. This is used for eliminating and detecting high level of impulsive noise. The median filter algorithms increase the quality of the filtered images without increasing the computational complexity of median filter algorithm. These algorithms try to detect the noisy pixels and adaptively filter only these noisy pixels. This proposed method helps to remove impulse noise and also maintained its image details. This method also helps to enhance its image quality. The result indicates that AWMF filter provides very less detection error rate and high restoration quality especially for high-level noise.

Ibrahem et al [15] presents a method for the removal of salt and pepper noise and considers $3 \times 3$ window for the removal of noise. If all the pixels are compared for the removal of noise then window size must be increased to $5 \times 5$ window. The proposed technique in this paper works efficient at a noise density of $97 \%$.

Ahmed, Faruk et al[16] in this work, adaptive iterative fuzzy mean filter has been presented which is used for de-noising images degraded by salt and pepper noise. Adaptive mean filter are used for image de-noising because they reduce the smoothing and preserve the image edges. There are a plenty sources of noise that affects the image. The result analysis indicates that the algorithm to be superior to state-of-the-art filters.

\section{PRoposed Methodology}

In the proposed technique buffering operations are performed with different window sizes. The buffered image is then pass to the median filter for the removal of salt and pepper noise. The proposed design is divided into two main blocks. In the first block row buffering technique is implemented and in the second block median filter is implemented. The blocks are described as:

Row Buffer: In the row buffering technique, 4 windowing operations are implemented in the proposed technique. In the first operation $3 \square 3$ window is implemented. In this window 3 inputs are loaded into the window and 9 outputs are generated after every clock cycle. Figure 1 shows the $3 \square 3$ input window operations. In the second window $4 \square 3$, four inputs are loaded into the buffer and after every clock cycle 12 outputs are generated. Figure 2 shows the $4 \square 3$ window operations. In the third window operation, $5 \square 3$ window operation is proposed. In this window operation 5 inputs are loaded into the buffer after every clock cycle and 15 outputs are generated which are further utilized for the median filtering calculation. Figure 3 shows the $5 \square 3$ window operation. And in the fourth operation, $5 \times 5$ window is implemented in which 5 inputs are loaded into the buffer and 25 outputs are generated for the median filter. Figure 4 shows the 5 X5 window operation.

\begin{tabular}{|l|l|l|l|l|l|l|}
\hline In7 & In8 & In9 & $\longrightarrow$ & & & \\
\hline In4 & In5 & In6 & I & & & \\
\hline In1 & In2 & In3 & $\longmapsto$ & & & \\
\hline & & & & & & \\
\hline & & & & & & \\
\hline & & & & & & \\
\hline
\end{tabular}

Fig.1. $3 \square 3$ Window Operation

\begin{tabular}{|l|l|l|l|l|l|l|}
\hline In10 & In11 & In12 & $\longrightarrow$ & & & \\
\hline In7 & In8 & In9 & $\longrightarrow$ & & & \\
\hline In4 & In5 & In6 & $\longrightarrow$ & & & \\
\hline In1 & In2 & In3 & $\longrightarrow$ & & & \\
\hline & & & & & & \\
\hline & & & & & & \\
\hline
\end{tabular}

Fig.2. $4 \square 3$ Window Operation

\begin{tabular}{|l|l|l|l|l|l|l|}
\hline In13 & In14 & In15 & $\longrightarrow$ & & & \\
\hline In10 & In11 & In12 & $\longrightarrow$ & & & \\
\hline In7 & In8 & In9 & $\longrightarrow$ & & & \\
\hline In4 & In5 & In6 & $\longrightarrow$ & & & \\
\hline In1 & In2 & In3 & $\longrightarrow$ & & & \\
\hline & & & & & & \\
\hline
\end{tabular}

Fig.3. $5 \square 3$ Window Operation

\begin{tabular}{|l|l|l|l|l|l|l|}
\hline In21 & In22 & In23 & In24 & In25 & $\longrightarrow$ & \\
\hline In16 & In17 & In18 & In19 & In20 & $\longrightarrow$ & \\
\hline In11 & In12 & In13 & In14 & In15 & $\longrightarrow$ & \\
\hline In6 & In7 & In8 & In9 & In10 & $\longrightarrow$ & \\
\hline In1 & In2 & In3 & In4 & In5 & $\longrightarrow$ & \\
\hline & & & & & & \\
\hline
\end{tabular}

Fig.4. 5X5 Window Operation

Median Filter: Median filtering operation is implemented for the removal of salt and pepper noise in the image. Median Filter operation is a convolution operation, which is implemented by sorting the rows first, and then sorting the columns and finally diagonal sorting is performed in a $3 \square 3$ window. Figure 4-6 shows the median filtering operation such as Row wise sorting, Column wise sorting and Diagonal sorting implemented in the proposed design.

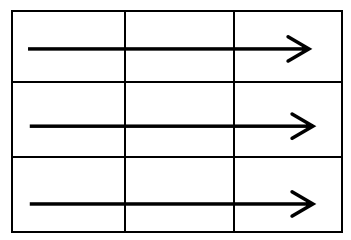

Fig.5. Row Wise Sorting

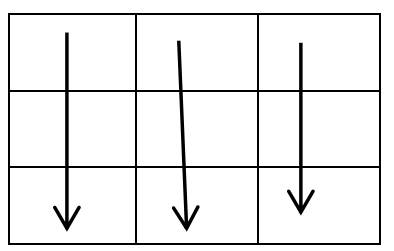

Fig.6. Column Wise Sorting 


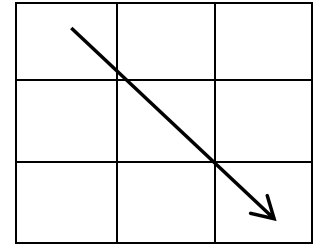

Fig.7. Diagonal Sorting

\section{RESULTS AND DISCUSSIONS}

The proposed methodology is implemented using the Xilinx Vertex 5 FPGA and the language used for the implementation is VHDL. The results for the number of resources used and the speed of operation are compared for the three different window operations for buffering. Table 1 shows the comparison of number of slices and other resources used and the total time required for the completion of buffering operations. The comparison table shows the total time required to buffer a $256 \times 256$ image with two rows zero padded in the image. The total time shows the speed of operation of buffering for different window operations. The table also shows the resources utilized by the FPGA for different window operations. The speed of operations for $5 X 5$ is increased as the total time is decreased with an increment in the area utilized. So a trade off must be maintained in order to efficiently buffer the image.

Table.1. Comparison table

\begin{tabular}{|l|l|l|l|l|l|}
\hline Parameters & 3 X3 Window & 4 X3 Window & 5 X3 Window & $\begin{array}{l}5 X 5 \text { Window } \\
\text { (Proposed) }\end{array}$ & 5X5 Window (Basic) \\
\hline No. of Slice Registers & 75 & 99 & 123 & 208 & 1296 \\
\hline No. of LUTs & 516 & 828 & 1151 & 3414 & 2400 \\
\hline No. of LUT-FF Pairs & 38 & 44 & 54 & 205 & 192 \\
\hline Latency (ns) & 19.514 & 18.883 & 18.907 & 19.674 & - \\
\hline Total Time (ns) & 221880 & 166410 & 133128 & 79876 & - \\
\hline
\end{tabular}

The resources utilized by the $5 \mathrm{X} 5$ window is compared to the base paper approach and it comes out that the proposed approach utilizes optimal resources as compared to basic approach. Figure 7 shows the simulation results of the proposed methodology for $3 \times 3$ window. Table 2 and Table 3 show the comparison of
PSNR and MSE with the base paper approach. Figure 8 (a-c) shows the introduction of noise in the image and the original image. The peak signal to noise ratio value is calculated for the noisy image with respect to the original image and is shown in table 2 and 3.
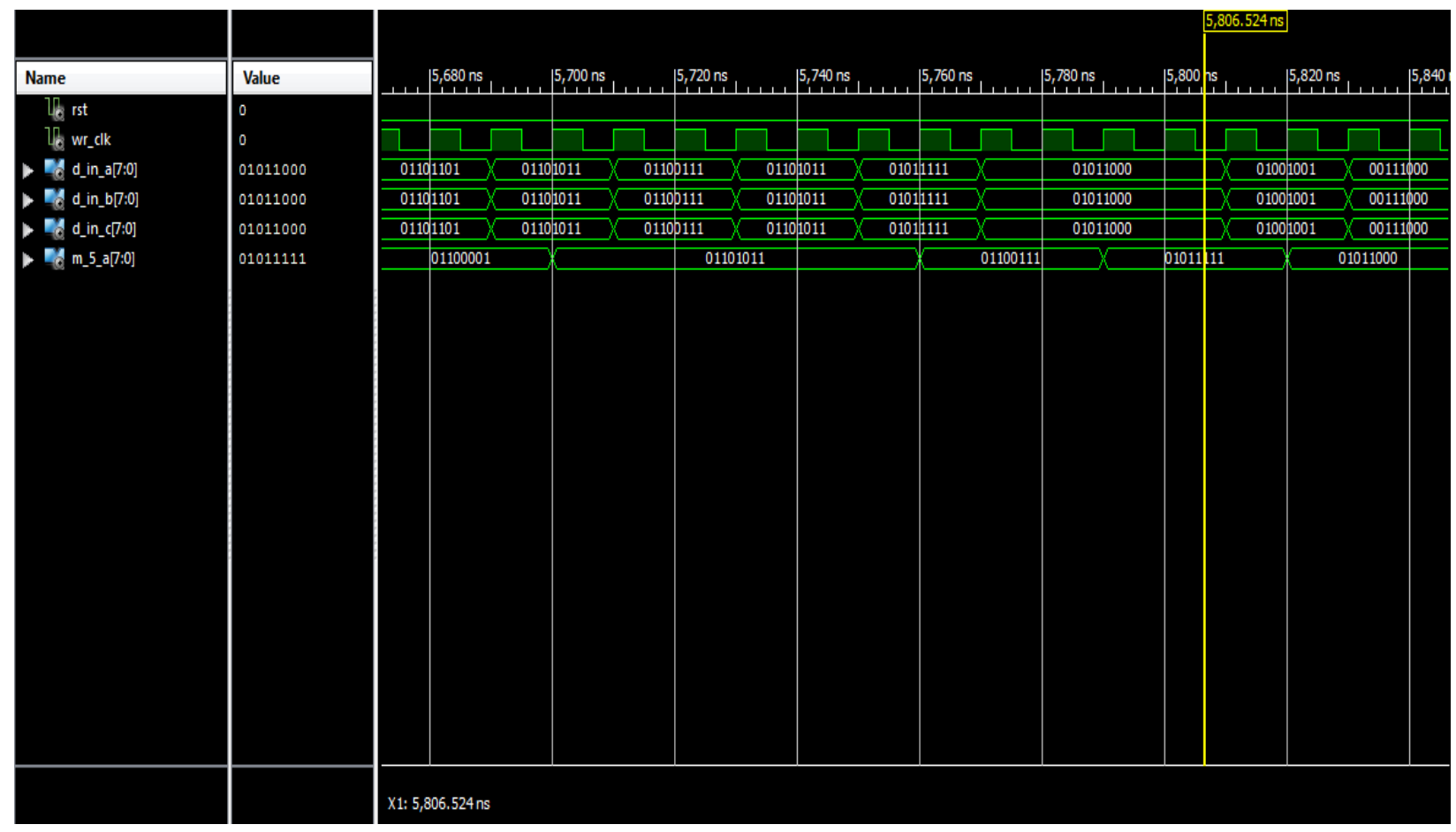

Fig.8. Simulation Results 
Table.2. Comparison Table PSNR with Base Paper Approach

\begin{tabular}{|c|c|c|c|c|}
\hline Image & $\begin{array}{c}\text { Basic Approach (3\% } \\
\text { noise) }\end{array}$ & $\begin{array}{c}\text { Proposed Approach } \\
(3 \% \text { noise })\end{array}$ & $\begin{array}{c}\text { Basic Approach } \\
(5 \% \text { noise })\end{array}$ & $\begin{array}{c}\text { Proposed Approach } \\
(5 \% \text { noise })\end{array}$ \\
\hline Lena & 25.54 & 28.5466 & 25.41 & 26.45 \\
\hline Baboon & 22.72 & 25.19 & 22.63 & 23.51 \\
\hline Peppers & 26.82 & 27.96 & 26.43 & 26.95 \\
\hline
\end{tabular}

Table 3 Comparison Table MSE with Base Paper Approach

\begin{tabular}{|c|l|l|l|l|}
\hline Image & $\begin{array}{l}\text { Basic Approach (3\% } \\
\text { noise) }\end{array}$ & $\begin{array}{l}\text { Proposed } \\
\text { Approach } \\
\text { (3\% noise) }\end{array}$ & $\begin{array}{l}\text { Basic } \\
\text { Approach } \\
\text { (5\% noise) }\end{array}$ & $\begin{array}{l}\text { Proposed } \\
\text { Approach (5\% } \\
\text { noise) }\end{array}$ \\
\hline Lena & 181.44 & 90.8698 & 186.77 & 147.33 \\
\hline Baboon & 347.5 & 205.35 & 354.72 & 300.88 \\
\hline Peppers & 135.07 & 113.9 & 147.6 & 134.95 \\
\hline
\end{tabular}
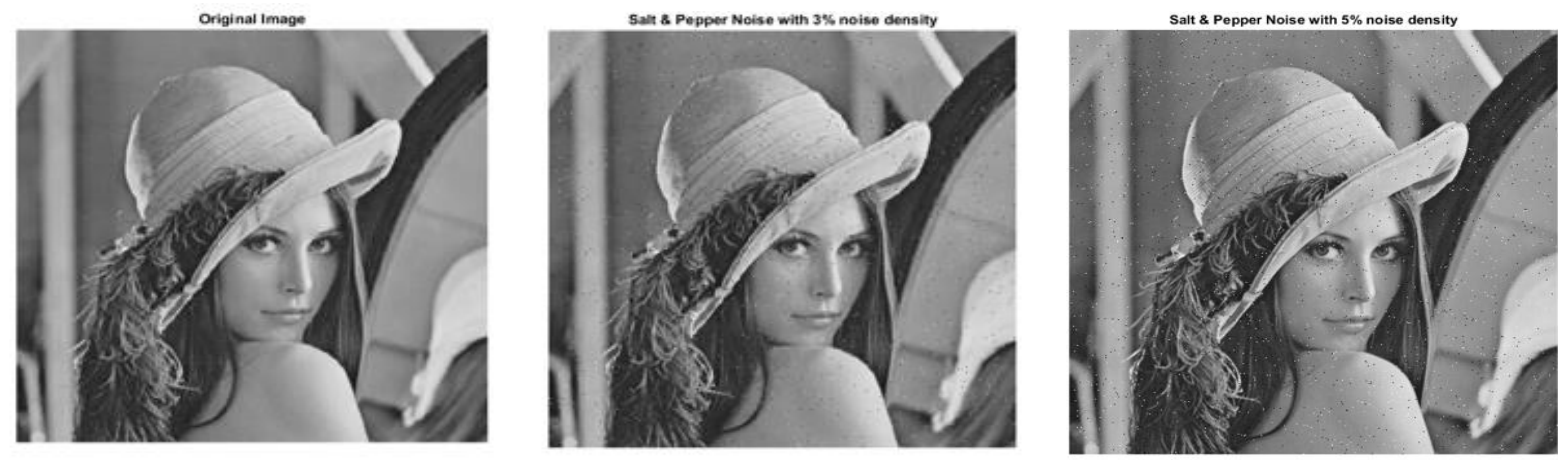

(a) Lena Image
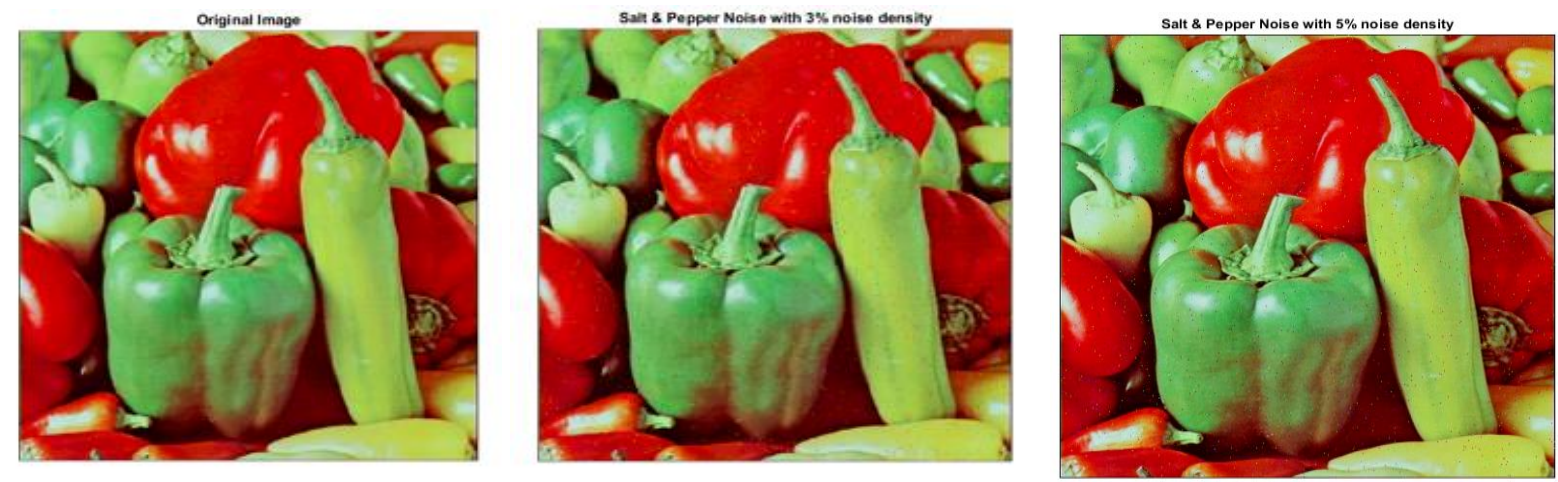

(b) Peppers Image
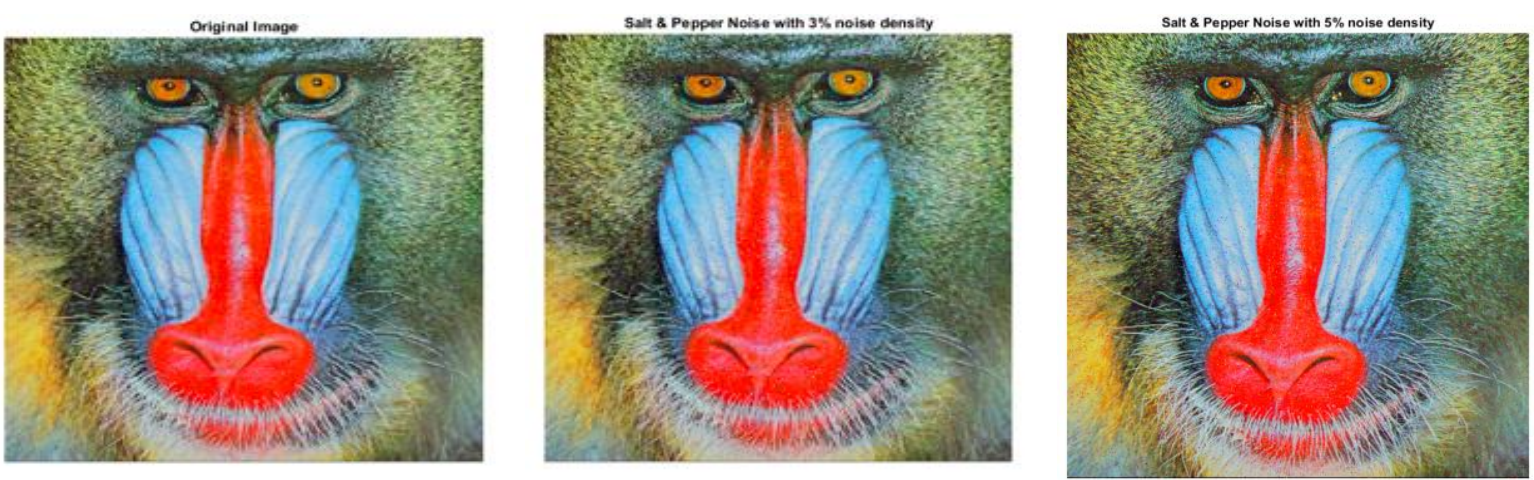

(c) Baboon Image

Fig.9. (a-c): Original Image and Noisy Image 


\section{CONCLUSION}

Buffering in digital image processing applications is an important area of concern in recent times. In the proposed technique four different sized windows are proposed for buffering the image and are compared on the basis of area and speed parameters. Also 5X5 window implemented here is compared to the base approach and shows an optimized value of the total area utilized by the device. It is clear from the proposed technique that as the number of rows in the buffering operation increases, the area utilized by the device also increases and to a larger extent. The speed of reading is increased but the memory requirement to hold and process the data also increases. The three test case images are considered and obtained a greater PSNR value and lesser MSE value as compared to the base approach. In future other window operation along with convolution filter like Adaptive Median filter must be implemented and used by changing the row and column values in Window size.

\section{ACKNOWLEDGMENT}

Authors would like to thank Chandigarh Engineering College (CEC) Landran, Mohali (India) for important and timely help in research. The work we present in this paper is completely supported by CEC, Landran.

\section{REFERENCES}

[1] Verma, Kesari, Bikesh Kumar Singh, and A. S. Thoke. "An Enhancement in Adaptive Median Filter for Edge Preservation." Procedia Computer Science48 (2015): 2936.

[2] Saleem, S. Abdul, and T. Abdul Razak. "An Effective Noise Adaptive Median Filter for Removing High Density Impulse Noises in Color Images. "International Journal of Electrical and Computer Engineering (IJECE) 6, no. 2 (2015).

[3] Chakravarthy, SR Sannasi, and S. A. Subhasakthe. "Adaptive Median Filtering with Modified BDND Algorithm for the Removal of High-Density Impulse and Random Noise." (2015).

[4] Kaur, Amanpreet, Rahul Malhotra, and Ravneet Kaur. "Performance evaluation of non-iterative adaptive median filter." In Advance Computing Conference (IACC), 2015 IEEE International, pp. 1117-1121. IEEE, 2015.

[5] Habib, Muhammad, Ayyaz Hussain, Saqib Rasheed, and Mubashir Ali. "Adaptive fuzzy inference system based directional median filter for impulse noise removal." AEU-International Journal of Electronics and Communications 70, no. 5 (2016): 689-697.

[6] Bhateja, Vikrant, Kartikeya Rastogi, Aviral Verma, and Chirag Malhotra. "A non-iterative adaptive median filter for image denoising." In Signal Processing and Integrated Networks (SPIN), 2014 International Conference on, pp. 113-118. IEEE, 2014.

[7] Meher, Saroj K., and Brijraj Singhawat. "An improved recursive and adaptive median filter for high density impulse noise." AEU-International Journal of Electronics and Communications 68, no. 12 (2014): 1173-1179.

[8] Shanmugavadivu, P., and P. S. Jeevaraj. "Laplace equation based Adaptive Median Filter for highly corrupted images." In Computer Communication and
Informatics (ICCCI), 2012 International Conference on, pp. 1-5. IEEE, 2012.

[9] Mukherjee, Manali, and Mausumi Maitra. "Reconfigurable architecture of adaptive median filterAn FPGA based approach for impulse noise suppression." In Computer, Communication, Control and Information Technology (C3IT), 2015 Third International Conference on, pp. 1-6. IEEE, 2015.

[10] Kalali, Ercan, and Ilker Hamzaoglu. "A low energy 2D adaptive median filter hardware." In Design, Automation $\&$ Test in Europe Conference \& Exhibition (DATE), 2015, pp. 725-729. IEEE, 2015.

[11] Hsieh, Mu-Hsien, Fan-Chieh Cheng, Mon-Chau Shie, and Shanq-Jang Ruan. "Fast and efficient median filter for removing 1-99\% levels of salt-and-pepper noise in images." Engineering Applications of Artificial Intelligence26, no. 4 (2013): 1333-1338.

[12] Sree, P. Syamala Jaya, Pradeep Kumar, Rajesh Siddavatam, and Ravikant Verma. "Salt-and-pepper noise removal by adaptive median-based lifting filter using second-generation wavelets." Signal, Image and Video Processing 7, no. 1 (2013): 111-118.

[13] Deka, Bhabesh, and Sangita Choudhury. "A multiscale detection based adaptive median filter for the removal of salt and pepper noise from highly corrupted images." International Journal of Signal Processing, Image Processing and Pattern Recognition 6, no. 2 (2013): 129-144.

[14] Zhang, Peixuan, and Fang Li. "A new adaptive weighted mean filter for removing salt-and-pepper noise." IEEE Signal Processing Letters 21, no. 10 (2014): 1280-1283.

[15] Ibrahem, Hani M. "An efficient and simple switching filters for removal of high density salt-and-pepper noise." International Journal of Image, Graphics and Signal Processing 5.12 (2013) in MECS

[16] Ahmed, Faruk, and Swagatam Das. "Removal of highdensity salt-and-pepper noise in images with an iterative adaptive fuzzy filter using alpha-trimmed mean." IEEE Transactions on fuzzy systems 22 , no. 5 (2014): 13521358.

\section{Authors' Profiles}

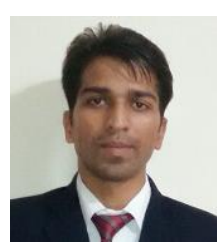

Arun Mahajan is a Post Graduate student of M.Tech VLSI at Chandigarh Engineering College Landran, Mohali, India. He completed his B.Tech degree in Electronics and Communication from SUS College of engineering and technology, Mohali, India. His area of interest includes Image Processing, VLSI, and digital communication.

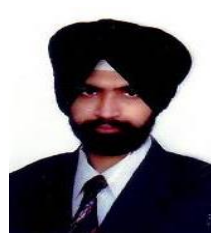

Mr. Paramveer Gill is an assistant professor in ECE Dept. at Chandigarh Engineering College Landran, Mohali, India. $\mathrm{He}$ is supervising many M.Tech students for their research work. His areas of interest are Image Processing, Wireless Sensor Network, Watermarking, Control and Automation. 
How to cite this paper: Arun Mahajan, Paramveer Gill,"2D Convolution Operation with Partial Buffering Implementation on FPGA", International Journal of Image, Graphics and Signal Processing(IJIGSP), Vol.8, No.12, pp.55-61, 2016.DOI: 10.5815/ijigsp.2016.12.07 